\title{
Análise do Perfil de Temperaturas no Gás de Exaustão de um Motor pelo Método das Diferenças Finitas
}

\author{
Cristiano H. G. Brito ${ }^{1}$, Guilherme F. Andrade ${ }^{2}$, José R. Sodré ${ }^{3}$ \\ Departamento de Engenharia Mecânica, Instituto Politécnico, PUC Minas \\ 30535-901, Belo Horizonte, MG \\ E-mail: cristianohbrito@globo.com ${ }^{1}$, gfandrade10@ hotmail.com², ricardo@pucminas.br ${ }^{3}$
}

\begin{abstract}
Resumo: $O$ conhecimento da energia transferida de calor é um quesito fundamental na análise, desenvolvimento e projeto de sistemas térmicos. A mensuração deste calor transferido pode ser feita empiricamente ou por simulação numérica, sendo a determinação dos perfis de temperatura uma etapa crucial para a resolução do problema de transferência de calor. Neste trabalho é realizado um estudo da transferência de calor no duto de exaustão de um motor de combustão interna. Para a determinação do perfil de temperaturas no gás de exaustão no interior do duto é implementado um algoritmo baseado no Método das Diferenças Finitas.
\end{abstract}

Palavras-chave: Transferência de Calor, Perfil de Temperaturas, Método das Diferenças Finitas, Duto de Exaustão, Motores de Combustão Interna.

\section{Introdução}

Embora os primeiros trabalhos acerca da transferência de calor em sistemas de exaustão tenham sido realizados há mais de 10 anos, este assunto continua atraindo a atenção devido à sua crucial importância na concepção de sistemas de exaustão modernos. De acordo com KANDYLAS (1998), tais estudos são importantes para a melhor compreensão destes sistemas, sendo capaz de influenciar, por exemplo, na transferência de calor pela estrutura, no aquecimento transiente do catalisador automotivo na partida a frio e no seu envelhecimento, ou no comportamento da regeneração de filtros de particulados. Para HEYWOOD (1981) é importante o conhecimento de informações quantitativas acerca da transferência de calor no sistema de exaustão, tanto para o projeto do motor quanto na previsão dos esforços aos quais o motor será submetido. De acordo com PLINT e MARTYR (1995) o sistema de exaustão é uma das fontes principais de perda de calor associado com o motor. Em motores com turbo-compressor, a energia perdida por transferência de calor pode ser particularmente significativa. Segundo MAVRIDOU (2010), a otimização da transferência de calor, nestes casos, pode ser obtida quer pelo aumento do coeficiente de fluxo de calor dos gases de exaustão, quer pelo aumento do volume de área específica de transferência de calor do trocador de calor. Entretanto, diversas abordagens para este problema podem ser encontradas na literatura.

\section{Formulação do Problema}

Para determinação do perfil de temperatura admite-se o modelo apresentado na Fig. 1, no qual considera-se a convecção interna entre as partículas do fluido e a convecção externa entre o fluido e o ambiente externo.

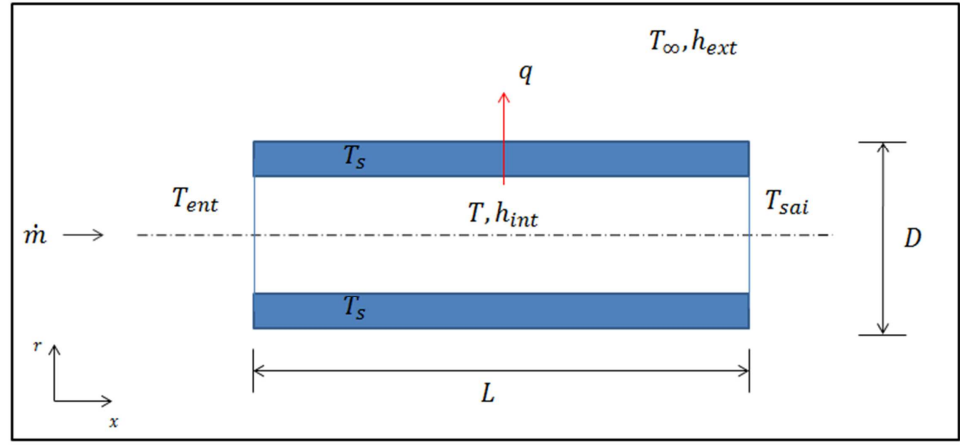

Figura 1: Modelo simplificado do duto de exaustão. 
Para determinação do perfil de temperatura do fluido no interior do duto seguem as seguintes etapas. Partindo de um modelo bidimensional, o escoamento do gás de exaustão no duto de exaustão pode ser inicialmente modelado como um escoamento laminar plenamente desenvolvido, em regime permanente, de um fluido com propriedades constantes. De acordo com INCROPERA (2011), para um tubo de comprimento não-nulo, desprezando-se a condução de calor na direção axial bem como a dissipação viscosa, tem-se a expressão geral para a convecção, que se aplica independentemente da natureza das condições térmicas na superfície e no escoamento no tubo.

$$
q_{\text {conv }}=\dot{m} c_{p}\left(T_{m, s a i}-T_{m, e m t r a}\right)
$$

Onde $q_{c o n v}$ é a taxa de transferência calor por convecção (W), $\dot{m}$ é a vazão mássica do gás de exaustão (kg/s) e $T_{m, e m t r a}$ e $T_{m, s a i}$ são as temperaturas médias da entrada e saída, respectivamente (K).

Segundo BEJAN (1995) uma equação diferencial que governa a distribuição de temperaturas pode ser determinada aplicando o balanço de energia no elemento diferencial anular da Figura 2. Desprezando os efeitos da condução axial liquida, a taxa de transferência de calor, q, é devida somente à condução através das superfícies radiais. A advecção de energia térmica ocorre somente na direção radial, uma vez que a velocidade radial do fluido é nula.

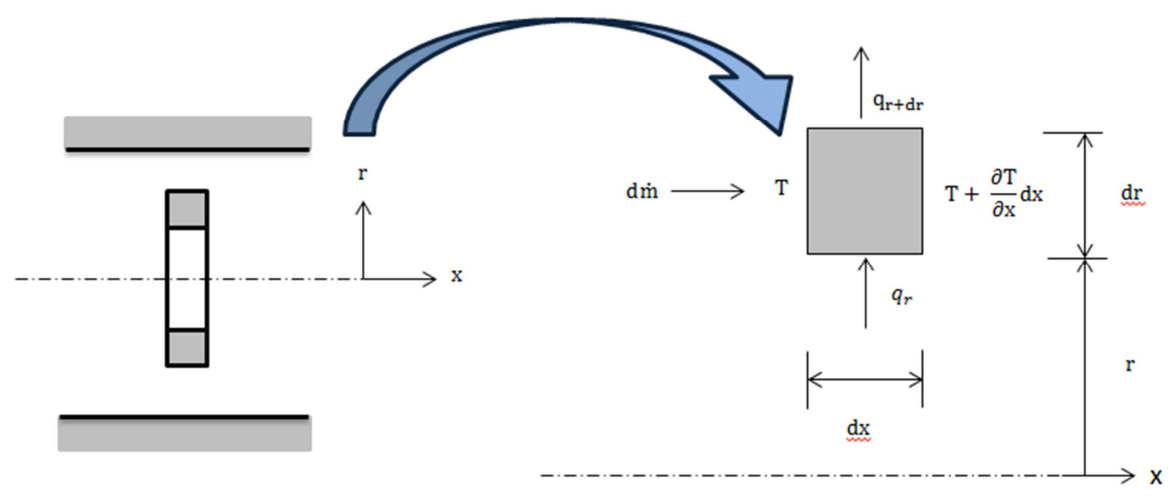

Figura 2: Balanço de energia térmica em um elemento diferencial no escoamento laminar plenamente desenvolvido em um duto circular.

$$
q_{r}-q_{r+d r}=(d \dot{m}) c_{p}\left[\left(T+\frac{\partial T}{\partial x} d x\right)-T\right] \therefore(d \dot{m}) c_{p} \frac{\partial T}{\partial x} d x=q_{r}-\left(q_{r}+\frac{\partial T}{\partial r} d r\right)=-\frac{\partial T}{\partial r} d r
$$

A vazão mássica na direção axial é

$$
d \dot{m}=\rho u 2 \pi r d r
$$

A taxa de transferência de calor na direção radial é

$$
q_{r}=-k \frac{\partial T}{\partial r} 2 \pi r d x
$$

Supondo propriedades constates, e substituindo as equações 3 e 4 na equação 2, tem-se a seguinte equação de equilíbrio:

$$
u \frac{\partial T}{\partial x}=\frac{\alpha}{r} \frac{\partial}{\partial r}\left(r \frac{\partial T}{\partial r}\right) \quad \therefore \frac{u}{\alpha} \frac{\partial T}{\partial x}=\frac{1}{r} \frac{\partial T}{\partial r}+\frac{\partial^{2} T}{\partial r^{2}}
$$


Onde $u$ é o perfil de velocidades do gás $(\mathrm{m} / \mathrm{s})$ e $\alpha$ é a difusividade térmica.

Para o escoamento laminar, plenamente desenvolvido, o perfil de velocidades assume a seguinte forma:

$$
u=u(r)=2 u_{m}\left[1-\left(\frac{r}{r_{0}}\right)^{2}\right]
$$

Onde a velocidade média do fluido $u_{m}$ pode ser expressa como a razão da vazão mássica $\dot{m}$ e da massa especifica $\rho\left(\mathrm{kg} / \mathrm{m}^{3}\right)$. Assim:

$$
u=u(r)=2 u_{m}\left[1-\left(\frac{r}{r_{0}}\right)^{2}\right]
$$

Segundo KAYS (2011), desconhecendo-se os coeficientes convectivos, pode-se determinar o perfil de temperatura na superfície a partir do principio das redes de resistências térmicas (Fig. 3).

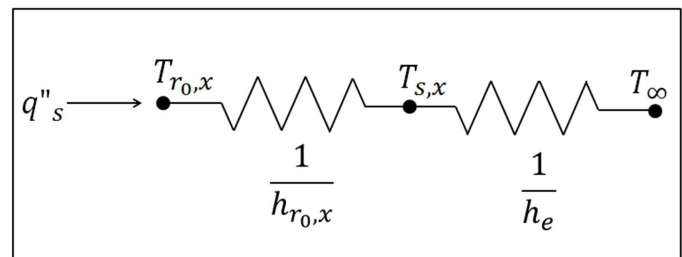

Figura 3: Rede de resistências térmicas

Assim, o fluxo térmico que atravessa a superfície pode ser expresso por:

$$
q^{\prime \prime}=\frac{T_{r_{0}, x}-T_{\infty}}{\frac{1}{h_{r_{0}, x}}+\frac{1}{h_{e}}}
$$

Onde, $h_{r_{0}, x}$ é o coeficiente convectivo do fluido na posição $\left(r_{0}, x\right) \mathrm{e}$ o $h_{e}$ é o coeficiente convectivo do ar externo $\left(\mathrm{W} / \mathrm{m}^{2} . \mathrm{K}\right)$. O coeficiente convectivo do fluido pode ser obtido a partir do número de Nuselt $(\mathrm{Nu})$ :

$$
N u_{D}=3,66+\frac{0,688\left(2 r_{0} / L\right) \operatorname{Re}_{D} \operatorname{Pr}}{1+0,04\left[\left(2 r_{0} / L\right) \operatorname{Re}_{D} \operatorname{Pr}\right]^{2 / 3}}
$$

Onde, $r_{0}$ é o raio do tubo (m), L é o comprimento avaliado (m), Pr é o número de Prandtl, e $\operatorname{Re}_{D}$ é o número de Reynolds para as condições da posição $\left(r_{0}, x\right)$. $\operatorname{Re}_{D}$ pode ser calculado como:

$$
\operatorname{Re}_{D}=\frac{2 \rho u r_{0}}{v}
$$

Definido o número de Nulset, pode-se obter o coeficiente convectivo $h_{r_{0}, x}$ a partir da seguinte relação:

$$
N u_{D}=\frac{2 h_{r_{0}, x} r_{0}}{k} \quad \therefore \quad h_{r_{0}, x}=N u_{D} \frac{k}{2 r_{0}}
$$


A partir do coeficiente convectivo do fluido, e conhecendo também o coeficiente convectivo do ar externo, pode-se determinar o fluxo de calor $q^{\prime \prime}{ }_{s}$ pela equação (8) e a partir do fluxo de calor determinar a temperatura da superfície como segue:

$$
q^{\prime \prime}{ }_{s}=h_{e}\left(T_{s, x}-T_{\infty}\right) \quad \therefore \quad T_{s, x}=\frac{q^{\prime \prime}}{h_{e}}+T_{\infty}
$$

\section{Metodologia}

A partir do método das diferenças finitas resolve-se a equação de equilíbrio (5) para obtenção do perfil de temperatura do fluido no interior do tubo (Fig. 4) e, a partir do perfil de temperaturas do fluido, determina-se o perfil na superfície pelo método da rede de resistências térmicas.

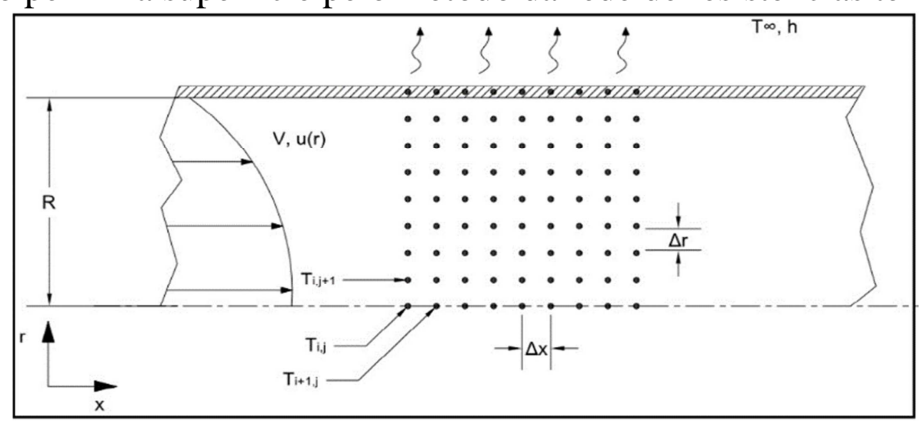

Figura 4: Rede nodal implementada no software.

A equação de equilíbrio na forma de diferenças finitas é apresentada a seguir

$$
\frac{u}{\alpha} \frac{\partial T}{\partial x}=\frac{1}{r} \frac{\partial T}{\partial r}+\frac{\partial^{2} T}{\partial r^{2}} \quad \therefore \quad \frac{u}{\alpha}\left(\frac{T_{i+1, j}-T_{i, j}}{\Delta x}\right)=\frac{1}{r}\left(\frac{T_{i+1, j}-T_{i, j}}{\Delta r}\right)+\left(\frac{T_{i, j-1}+T_{i, j+1}-2 T_{i, j}}{(\Delta r)^{2}}\right)
$$

Para o perfil de temperaturas da superfície considera-se a seguinte expressão:

$$
T_{s, j}=\frac{1}{h_{e}}\left[\frac{T_{r_{0}, x}-T_{\infty}}{\frac{1}{h_{r_{0}, x}}+\frac{1}{h_{e}}}\right]+T_{\infty}
$$

Onde o coeficiente convectivo $h_{r_{0}, x}$ é obtido a partir dos números de Nusselt e Reynolds, substituindo as equações (10) e (9) na equação (11). Para o cálculo, adotou-se as dimensões e características de um grupo motor-gerador operando em determinadas cargas nominais. A Tabela 1 mostra as características do motor diesel adotado. A Figura 5 mostra o fluxograma do algoritmo desenvolvido.

\begin{tabular}{|l|c|}
\hline PARÂMETRO & TIPO OU VALOR \\
\hline Tipo de construção & Diesel -4 tempos em linha \\
\hline Tipo de injeção & Direta \\
\hline Diâmetro x curso & $102 \times 120 \mathrm{~mm}$ \\
\hline Cilindrada unitária & 0,980 litros \\
\hline Número de cilindros & 4 \\
\hline Cilindrada total & 3,922 litros \\
\hline Tipo de Aspiração & Natural \\
\hline
\end{tabular}

Tabela 1: Características do motor 
INícıo

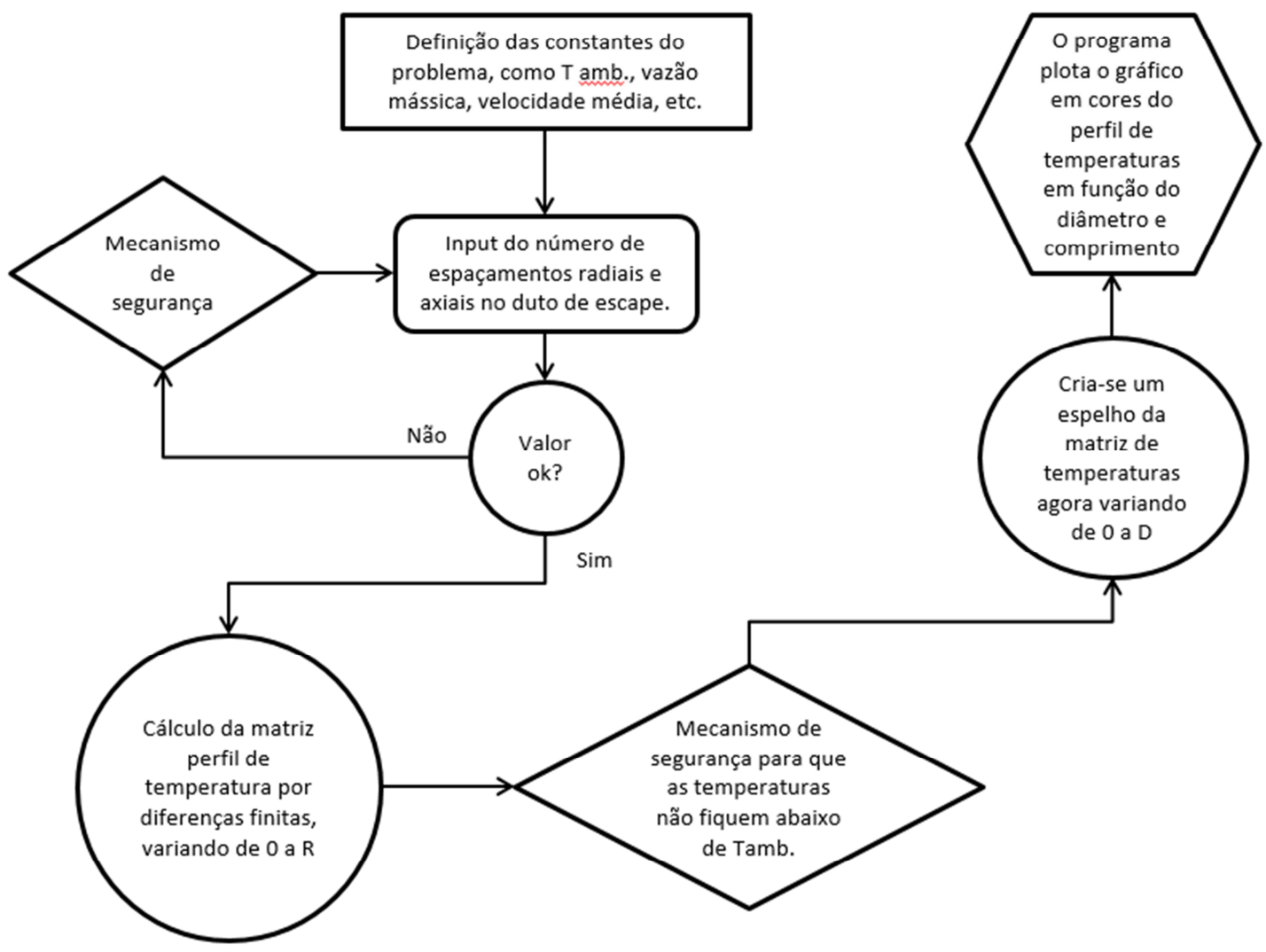

Figura 4: Fluxograma do algoritmo implementado

Todo o cálculo numérico foi realizado com a versão 32 bits do software Matlab® (2012) em um micro computador com processador Intel ${ }^{\circledR}$ Core 2 Duo $^{\mathrm{TM}} \mathrm{CPU}$ T6570 @ 2,10 GHz com 4GB de memória.

\section{Resultados}

A partir da metodologia empregada e utilizando o valor mensurado do duto de exaustão do motor e os parâmetros de operação descritos por MORAIS et al. (2013) (Tab. 2), obteve-se os seguintes resultados mostrados na Fig. 5. A Figura 5 mostra que as temperaturas mais elevadas do gás de exaustão se propagam mais distante ao longo do duto com o aumento da carga aplicada ao motor.

\begin{tabular}{|l|c|c|c|c|c|}
\hline VARIÁVEL MEDIDA & UNIDADE & \multicolumn{4}{|c|}{ VALORES OBSERVADOS } \\
\hline CARGA nominal & $\mathrm{KW}$ & $\mathbf{0}$ & $\mathbf{1 0}$ & $\mathbf{2 0}$ & $\mathbf{4 0}$ \\
\hline Temperatura Ambiente & ${ }^{\circ} \mathrm{C}$ & 27 & 27 & 27 & 27 \\
\hline $\begin{array}{l}\text { Temperatura de Entrada } \\
\text { do Gás de Exaustão }\end{array}$ & ${ }^{\circ} \mathrm{C}$ & 149,4 & 239,37 & 332,12 & 598,27 \\
\hline $\begin{array}{l}\text { Vazão Mássica dos } \\
\text { Gases de Exaustão }\end{array}$ & $\mathrm{kg} / \mathrm{h}$ & 148,44 & 144,71 & 142,72 & 132,95 \\
\hline Peso específico & $\mathrm{kg} / \mathrm{m}^{3}$ & 1,06 & 1,06 & 1,06 & 1,06 \\
\hline Calor especifico & $\mathrm{J} /(\mathrm{kg} . \mathrm{K})$ & 1015 & 1034 & 1054 & 1114 \\
\hline Viscosidade & $1 \mathrm{E} 6$. Pa.s & 29,18 & 40,31 & 53,26 & 98,06 \\
\hline Condutividade Térmica & $\begin{array}{c}1 \mathrm{E} 3 . \\
\mathrm{W} /(\mathrm{m} . \mathrm{K})\end{array}$ & 35,03 & 41,76 & 47,56 & 60,26 \\
\hline Diametro do tubo & $\mathrm{mm}$ & 66 & 66 & 66 & 66 \\
\hline
\end{tabular}

Tabela 2: Dados obtidos 

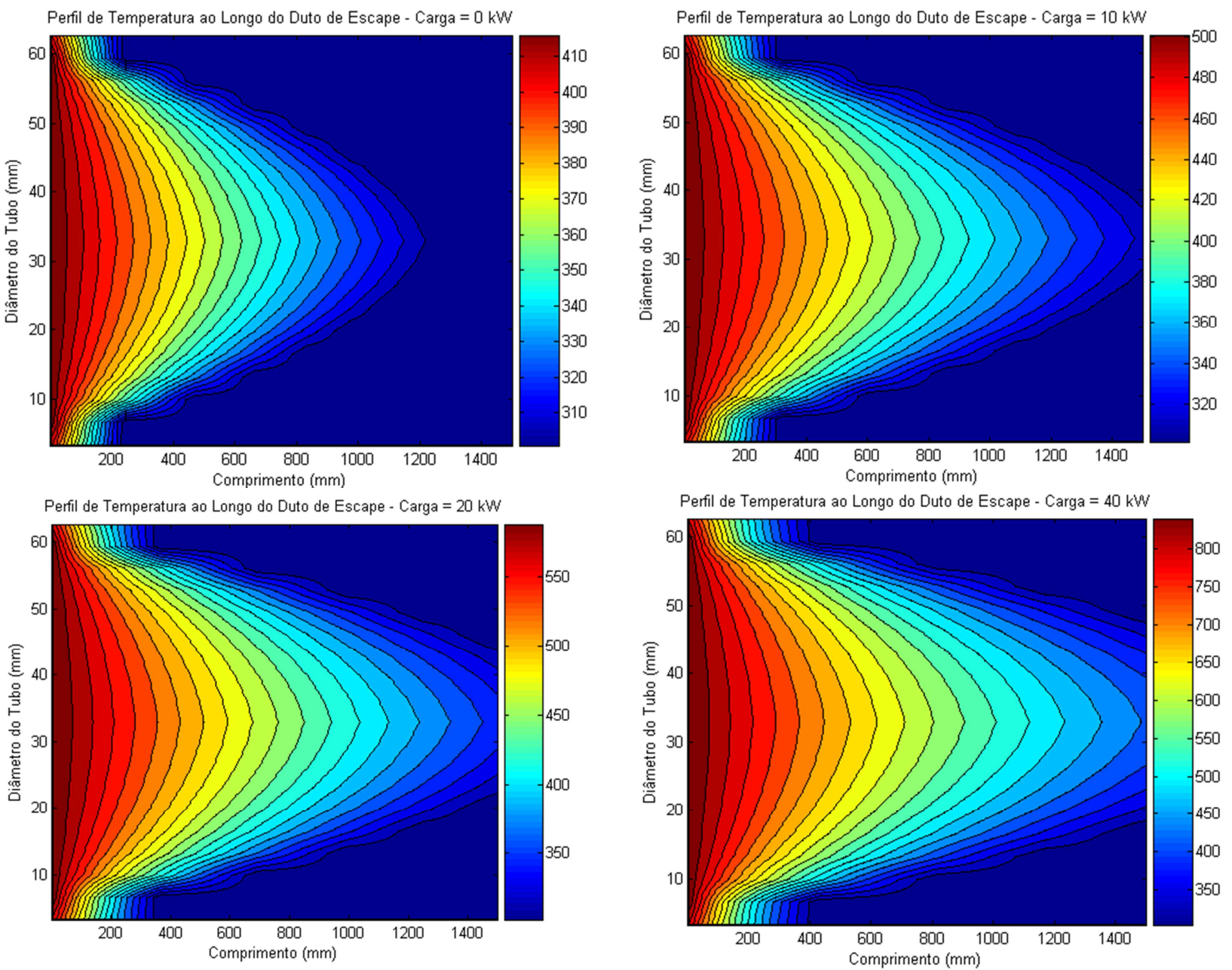

Figura 5: Perfis de temperatura obtidos paras as cargas de 0, 10, 20 e $40 \mathrm{~kW}$.

\section{Conclusão}

Foi realizado um estudo sobre a transferência de calor no duto de exaustão de um motor de combustão interna de um grupo motor-gerador, com o objetivo de determinar o perfil de temperaturas no duto utilizando o método das diferenças finitas. Implementou-se um algoritmo no software Matlab ® para resolução das equações e obtenção dos perfis de temperatura para diferentes cargas aplicadas ao motor. Os perfis de temperatura obtidos mostram que a medida que se aumenta a carga aplicada no motor, se estende o gradiente de temperatura ao longo do comprimento do tubo, como resultado do aumento da temperatura do gás de exaustão.

\section{Referências}

[1] A. Bejan, Convection Heat Transfer, 3. Ed, Wiley, 2004. p 108-158.

[2] J. Heywood, An Experimental and Analytical Study of Heat Transfer in an Engine Exhaust Port, Int. J. Heat Mass Transfer, Grã-Bretanha, 24 (1981) 581-595.

[3] I. Kandylas, A Engine exhaust system design based on heat transfer computation, Energy Conversion \& Management, 40 (1999) 1057-1072.

[4] W. Kays, Convective Heat and Mass Transfer, 3. Ed, McGraw-Hill, 1993. p 96-144.

[5] S. Mavridou, Comparative design study of a diesel exhaust gas heat exchanger for truck applications with conventional and state of the art heat transfer enhancements, App. Ther. Eng., 30 (2010) 935-947.

[6] M. Plint, A. Martyr, Engine Testing, Butterworth-Heinemann, 1995.

[7] A. Morais, M. Justino, O. Valente, S. Hanriot, J. Sodré, Hydrogen impacts on performance and

$\mathrm{CO}_{2}$ emissions from a diesel power generator, Int. J. Hydrogen Energy, 38 (2013) 6857-6864. 